\title{
Does Aspartate Aminotransferase to Alanine Aminotransferase Ratio Predict Acute Kidney Injury After Cardiac Surgery?
}

\author{
Gültekin Yıldırım, MD, ${ }^{1}$ Bolat Ali, MD,${ }^{1}$ Keles Hatice, $M D,{ }^{2}$ Tekeli Kunt Atike, $M D, \mathrm{PhD}^{1}$ \\ ${ }^{1}$ Department of Cardiovascular Surgery, Kirikkale University Medical School, Kirikkale, Turkey; ${ }^{2}$ Department of Internal Medicine, \\ Kirikkale University Medical School, Kirikkale, Turkey
}

\section{ABSTRACT}

Background: Aspartate aminotransferase (AST) to alanine aminotransferase (ALT) ratio (AST/ALT) frequently is used in the diagnosis and prognosis of liver diseases, however it is also used in the diagnosis and prognosis of many other diseases, such as myocardial infarction, acute ischemic stroke, and peripheral artery disease. Acute kidney injury (AKI) is one of the most important complications after cardiac surgery and is one of the main causes of morbidity and mortality. The purpose of the study was to analyze the relationship between AST to ALT and AKI after isolated coronary artery bypass graft surgery (CABG).

Methods: We retrospectively reviewed the prospectively collected data of 253 adult patients, who underwent isolated CABG surgery with normal renal function (baseline serum creatinine value $<1.4 \mathrm{mg} / \mathrm{dL}$ ). Preoperative (T0) and postoperative day 1 and day 3 (T1 and T2) serum AST and ALT levels were analyzed, and AST/ALT was calculated. A preoperative AST/ALT of 1.22 was found to be the best cutoff point for predicting postoperative AKI. Kidney injury was interpreted, according to RIFLE classification. The effect of AST to ALT ratio on AKI after CABG was determined using logistic regression analysis, and the results were expressed as odds ratio (OR) with a $95 \%$ confidence interval $(\mathrm{CI})$. A $P$ value $<.05$ was considered statistically significant.

Results: Postoperative AKI occurred in 40 patients (15.8\%). On logistic regression analysis, higher AST/ALT both preoperatively and postoperatively were associated with an increased incidence of postoperative AKI (T0: OR, 3.983; 95\% CI, 1.940-8.180, $P<.001$, T1: OR, 2.760; 95\% CI, 1.381-5.515, $P=.004$, T2: OR, 2.515; 95\% CI, 1.195-5.294, $P=.015)$.

Conclusion: Preoperative and postoperative elevated AST to ALT ratio seems to be associated with an increased incidence of AKI after elective isolated CABG surgery.

Received March 30, 2021; accepted April 8, 2021.

Correspondence: Atike Tekeli Kunt, MD, PhD, Professor of Cardiovascular Surgery, Kirikkale University Medical School, Yenisebir, Ankara Yolu 7.Km. Kirıkkale Üniversitesi Kampïsü, 71450 Yabsiban/Kirikkale; +90-532-74678-69 (e-mail: atikemd@gmail.com).

\section{INTRODUCTION}

Transaminases, namely aspartate aminotransferase (AST) and alanine aminotransferase (ALT) are enzymes found mainly in the liver, but also found in red blood cells, heart cells, muscle tissue, pancreas, and kidneys [Botros 2013]. Although AST to ALT ratio (AST/ALT) frequently is used in the diagnosis and prognosis of liver diseases, it also is used in the diagnosis and prognosis of many other diseases, such as acute ischemic stroke, myocardial infarction, and peripheral artery disease [Steininger 2018; Gao 2107; Rief 2016]. After open heart surgery, acute kidney injury (AKI) is a well-known risk factor for the development of serious complications that increase mortality and morbidity and increase healthcare costs [Yeo 2008]. A thorough understanding of the risk factors and mechanisms underlying the development of AKI may help in identifying useful biomarkers and designing preventive methods. Age, diabetes mellitus (DM), preexisting renal dysfunction, hypertension, impaired left ventricular function, and severe arteriosclerosis are the major risk factors for the development of AKI [Weerasinghe 2001; Chertow 1997]. Intraoperative renal hypoperfusion, nonpulsatile flow, inflammatory response syndrome due to cardiopulmonary bypass and genetic polymorphisms are also important causative factors of AKI [Suen 1998; Hall 1997; Isbir 2007]. Serum AST and ALT also are related with oxidative stress and systemic inflammation, and they are known as good predictors of tissue injury, mainly liver injury [Sheth 1998]. The purpose of the current study was to analyze the relationship between AST/ALT and AKI after isolated CABG.

\section{MATERIALS AND METHOD}

This single-center, retrospective study was carried out at Kırıkkale University Faculty of Medicine Hospital between January 2014 and January 2017. Written informed consent was obtained from each patient before the operation. The study protocol was approved by the Kirkkale University Faculty of Medicine Ethics Committee (decision number: 2020.06.16). The study was conducted in accordance with the principles of the Declaration of Helsinki.

\section{Patients}

After we received institutional review board approval, we retrospectively reviewed the prospectively collected data of 
Table 1. RIFLE Classification [Bellomo 2004]

\begin{tabular}{|c|c|c|}
\hline Risk & Increased plasma creatinine $\times 1.5$ & $<0.5 \mathrm{~mL} / \mathrm{kg}^{-1} / \mathrm{h}^{-1} \times 6$ hours \\
\hline Failure & Increased plasma creatinine $x 3$ or acute plasma creatinine $\geq 350 \mu \mathrm{mol} / \mathrm{L}$ or acute rise $\geq \mu \mathrm{mol} / \mathrm{L}$ & $<0.3 \mathrm{~mL} / \mathrm{kg}^{-1} / \mathrm{h}^{-1} \times 24$ hours or anuria $\times 12$ hours \\
\hline Loss & Persistent acute renal failure $=$ complete loss of kidney function $>4$ weeks & \\
\hline
\end{tabular}

ESKD indicates end-stage kidney disease; GFR, glomerular filtration rate.

253 adult patients who underwent isolated CABG surgery with normal renal function (baseline serum creatinine value $<1.4 \mathrm{mg} / \mathrm{dl}$ ) from January 2014 to January 2017. All patients previously had granted permission for the use of their medical records for research purposes. The clinical data of the patients included demographic data, laboratory data, length of stay, in-hospital complications, and mortality.

\section{Laboratory assessment}

In addition to preoperative serum levels of ALT and AST (T0), postoperative day 1 and day 3 (T1 and T2) serum AST and ALT levels, the data of complete blood count (CBC), $\gamma$-glutamyl transferase (GGT), albumin, total bilirubin, total and high density lipoprotein cholesterol and serum levels of triglycerides also were recorded and AST/ALT was calculated. Patients with a history of chronic liver disease, drug induced liver injury, a history of viral hepatitis and cirrhosis of any etiology, and serum aminotransferase levels higher than three times the upper limit of the reference range for the local laboratory, patients who were on either hemodialysis or peritoneal dialysis, patients with emergent surgery, recent myocardial infarction, peripheral vascular disease, and patients undergoing operations other than or in conjunction with CABG, were excluded from the study. An automatic biochemical analyzer, BS800M (Mindray, Bio-Medical Electronics Co. Ltd., Nanshan, Shenzhen, P.R. China), was used to measure the levels of AST, ALT, and other biochemical analysis. An automated blood analyzer, BC-6800 (Mindray, Bio-Medical Electronics Co. Ltd., Nanshan, Shenzhen, P.R. China), was used to measure CBC. The primary outcome was the development of AKI. Kidney injury was interpreted, according to RIFLE classification [Bellomo 2004], explained as RIFLE R: risk; RIFLE I: injury; RIFLE F: failure; RIFLE L: loss; and RIFLE E: end-stage kidney disease (Table 1).

\section{CABG procedure}

All operations were performed in a standardized approach by a Terumo roller pump (Terumo Advanced Perfusion System 1, Terumo ${ }^{\circledR}$, Ann Arbor, Michigan, USA) and membrane oxygenators (Inspire 8, LivaNova Sorin Group, Modena, Italy). Mild to moderate $\left(28-32^{\circ} \mathrm{C}\right)$ hypothermia and pulsatile flow of 2.2-2.4 L/m² were used. Myocardial protection was achieved with tepid antegrade blood cardioplegia, and a "hot shot" (250-500 ml) was delivered just before the removal of the aortic cross-clamp. The perfusion pressure was kept over $70 \mathrm{mmHg}$ at all times. Induction and maintenance of general anesthesia with endotracheal intubation were standardized in all the patients (fentanyl, midazolam, and isoflurane in oxygen with air). The same surgical team performed all of the operations.

Postoperative management: Postoperatively patients were followed in the intensive care unit (ICU), according to protocols of our institution. Electrocardiography, systemic mean arterial pressure, central venous pressure, pulmonary artery and wedge pressures, cardiac output and index, arterial blood gases, chest tube output, and hourly urine output were monitored. Serum electrolytes were measured in conjunction with arterial blood gas measurement. Fluid and electrolyte imbalances immediately were corrected with appropriate management. Hematocrit values $<25 \%$ were corrected with erythrocyte suspension administration postoperatively. Daily ALT, AST, blood urea nitrogen, serum and urea creatinine and serum electrolytes were measured uniformly in all patients until discharge from the hospital. AST/ ALT was calculated on postoperative day 1 (T1) and postoperative day 3 (T2) in addition to preoperative (T0) levels. Preoperative and postoperative creatinine clearances and peak creatinine clearance were calculated, according to the formulations reported in the literature [Lassnigg 2000; Cockcroft 1976]. The indication criteria for RRT, hyperkalemia ( $>6 \mathrm{mmol} / \mathrm{l}$ ), oliguria $<0.5 \mathrm{ml} /$ $\mathrm{kg} / \mathrm{h}$ for 12 hours or anuria, and metabolic acidosis, were determined by our nephrologist. Vascular access for RRT was with a dual lumen catheter via a central venous vein. Patients were heparinized to achieve activated clotting time of 200 seconds. Fresenius polysulfone filter (Fresenius Medical care AG, Bad Hamburg, Germany) was used for filtration.

\section{Statistical analysis}

All statistics were performed using SPSS version 18.0 for Windows (IBM Corporation, New York, USA). Continuous variables were expressed as mean $\pm \mathrm{SD}$ and were compared by unpaired Student's t-test or the chi-squared test. The effect of AST/ALT on AKI after CABG was determined using logistic regression analysis and the results were expressed as odds ratio (OR) with a $95 \%$ confidence interval (CI). Sensitivity of the AUCs for prediction of postoperative AKI based on RIFLE criteria was calculated using the DeLong's method. 




Figure 1. ROC curve of predicting postoperative AKI with preoperative AST to ALT ratio (TO).



Figure 2. ROC curve of predicting postoperative AKI with postoperative AST to ALT ratio on day 1 (T1).

The best cutoff point of AST/ALT was obtained using the Youden index. Patients were then divided into two groups according to the best cutoff point of preoperative AST/ALT, including a high AST/ALT $(\geq 1.22)$ group (Group I) and a low AST/ALT ( $<1.22)$ group (Group II). A $P$-value $<.05$ was considered statistically significant.

\section{RESULTS}

Patient demographics and perioperative data are shown in Table 2. The receiver operating characteristic (ROC) curve showed that the preoperative ASL/ALT ratio could predict AKI after CABG. The area under the curve (AUC) was 0.762 $(P<.001)$ with a $95 \%$ confidence interval of $0.704-0.813$ with a best cut-off value of 1.22 , sensitivity of $80 \%$ and specificity of $68.5 \%$ (Figure 1). There were 100 patients $(39.5 \%)$ in Group I with AST/ALT $\geq 1.22$, and 153 patients $(60.5 \%)$ in Group II with AST/ALT $<1.22$. Among the patient characteristics and perioperative data, female sex, and AST/ALT significantly were higher in Group I, whereas body mass

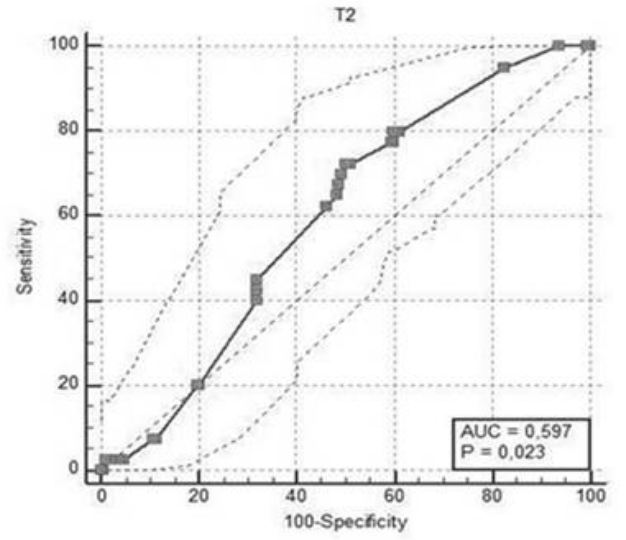

Figure 3. ROC curve of predicting postoperative AKI with postoperative AST to ALT ratio on day 3 (T2).

index (BMI $>30 \mathrm{~kg} / \mathrm{m} 2)$ and metabolic syndrome (MetS) were found to be higher in Group II. Other data did not show statistically significant differences between the groups. Postoperative AKI occurred in 40 patients $(15.8 \%)$ in total, 27 patients $(27 \%)$ in Group I and 13 patients $(8.5 \%)$ in Group II. On logistic regression analysis, the presence of high AST/ ALT preoperatively (T0) was shown to be associated with an increased incidence of postoperative AKI (OR, 3.983; 95\% CI, 1.940-8.180; $P<.001)$. Additionally, the ROC curve analysis showed that the ASL/ALT ratio on the postoperative day 1 (T1) could predict AKI after CABG. AUC was 0.612 $(P=.029)$ with a $95 \%$ confidence interval of $0.549-0.673$ with a best cut-off value of $>1.74$, sensitivity of $60 \%$ and specificity of $65.3 \%$ (Figure 2). The ROC curve analysis also showed that the ASL/ALT on the postoperative day 3 (T2) could predict postoperative AKI. AUC was $0.597(P=.023)$ with a $95 \%$ confidence interval of $0.533-0.658$ with a best cut-off value of $>1.21$, sensitivity of $72.5 \%$ and specificity of $49.8 \%$ (Figure 3). Multivariate logistic regression analysis revealed that DM (OR, 3.348; 95\% CI, 1.650-6.799; $P=.001$ ), obesity (BMI $\left.>30 \mathrm{~kg} / \mathrm{m}^{2}\right)(\mathrm{OR}, 2.885 ; 95 \% \mathrm{CI}, 1.435-5.798 ; P<.001)$, and MetS (OR, 4.195; 95\% CI, 2.068-8.509; $P=.003$ ) were other independent risk factors for AKI after isolated CABG.

The preoperative mean serum creatinine was $0.89 \pm$ $0.19 \mathrm{mg} / \mathrm{dL}$ in Group I and $0.90 \pm 0.21 \mathrm{mg} / \mathrm{dL}$ in Group II $(P=.839)$. Postoperative peak serum creatinine levels were higher in Group I patients than in Group II $(1.48 \pm 0.89 \mathrm{mg} /$ $\mathrm{dL}$ and $1.23 \pm 0.87 \mathrm{mg} / \mathrm{dL}$, respectively, $P=.031)$. When results were compared according to the RIFLE stage, 27 patients in Group I included seven patients (26\%) in RIFLE R, 11 patients $(40.7 \%)$ in RIFLE I, and nine patients $(33.3 \%)$ in RIFLE F stage. Whereas 13 patients in Group II included nine patients $(69.2 \%)$ in RIFLE R and four patients (30.8\%) in RIFLE I; none of the patients in Group II were in RIFLE F stage (RIFLE $\mathrm{R}, P=.794$; RIFLE I, $P=.0012$; RIFLE F, $P<.001$ respectively). Additionally, we compared the results of AST/ALT on postoperative day 1 (T1) and day 3 (T2) and RIFLE stage that higher AST to ALT ratio was significantly associated with AKI in the RIFLE F stage $(P<.001$ and $P=.004$, respectively). 
Table 2. Baseline and perioperative characteristics of patients

\begin{tabular}{lccc}
\hline Clinical characteristics & $\begin{array}{c}\text { Group } \text { I }^{*} \\
(\mathrm{~N}=100)\end{array}$ & $\begin{array}{c}\text { Group II } \\
(\mathrm{N}=153)\end{array}$ & $P \ddagger$ \\
\hline Age, years & $60.2 \pm 11.3$ & $62.1 \pm 10.6$ & .182 \\
Female, \% & 52 & 24.8 & $<.001$ \\
Body mass index, >30 kg/m², \% & 30 & 47.1 & .009 \\
Hypertension, \% & 66 & 62.1 & .593 \\
Dyslipidemia, \% & 52 & 60.8 & .194 \\
Diabetes, \% & 33 & 37.9 & .503 \\
Metabolic syndrome, \% & 21 & 36.6 & .012 \\
LV function, \% & $54.7 \pm 8.5$ & $54.8 \pm 9.1$ & .960 \\
CPB time, min & $114.2 \pm 38.1$ & $112.0 \pm 36.7$ & .639 \\
Cross-clamp time, min & $65.5 \pm 23.5$ & $63.3 \pm 22.9$ & .456 \\
Serum creatinine, $\mathrm{mg} / \mathrm{dl}$ & $0.89 \pm 0.19$ & $0.90 \pm 0.21$ & .839 \\
Creatinine clearance, $\mathrm{ml} / \mathrm{min}$ & $93.2 \pm 37.5$ & $87.7 \pm 38.3$ & .332 \\
AST/ALT & $1.4 \pm 0.2$ & $1.0 \pm 1.1$ & $<.001$ \\
\hline
\end{tabular}

Data are presented as mean \pm SD where indicated. CPB, cardiopulmonary bypass; LV, left ventricle, AKI, acute kidney injury; RIFLE R: risk; I: injury; F: failure; L: loss; and E: end-stage kidney disease, AST, aspartate aminotransferase; ALT, alanine aminotransferase; ICU, intensive care unit. *Group I: patients with preoperative AST/ALT $\geq 1.22$, †Group II: patients with preoperative AST $/ A L T<1.22$. $\ddagger$ Chi-square and unpaired Student $t$ tests. Values in bold are statistically significant $(P<0.05)$.

Renal replacement therapy was used in $1.98 \%(\mathrm{~N}=5)$ of patients (all patients were in Group I). The creatinine value before the commencement of RRT was $3.86 \pm 0.49 \mathrm{mg} / \mathrm{dL}$. RRT was started 36-56 hours after surgery and used for a maximum of 7 days. The mean creatinine level was $1.32 \pm$ $0.74 \mathrm{mg} / \mathrm{dL}$ before hospital discharge and none of the patients became hemodialysis dependent.

The mean ICU time was $59.03 \pm 30.20$ hours in Group I and $48.56 \pm 23.16$ hours in Group II $(P=.002)$; in-hospital stay time was $7.65 \pm 2.41$ days in Group I and $7.04 \pm 1.51$ days in Group II $(P=.015)$. The intra-aortic balloon pump (IABP) support was required in $5.53 \%$ of patients $(10$ patients in Group I and four patients in Group II, $P=.021)$. Prolonged ventilatory support was necessary for $4.2 \%$ of patients and the mean ventilatory support time was $9.73 \pm 12.04$ in Group I and $7.15 \pm 2.92$ in Group II, $P=.012$ ) and none of these patients required tracheotomy. The 30-day mortality was 3.6\% $(\mathrm{N}=9$, seven patients in Group I and two patients in Group II, $P=.031$ ). All patients died due to low cardiac output and multiorgan failure, and these patients required RRT.

\section{DISCUSSION}

In cardiac surgery, postoperative acute renal damage is seen in $3-30 \%$ of patients, while damage requiring dialysis treatment develops in 1-5\% of patients [Brown 2007]. In the present study, we analyzed the association between preoperative and postoperative AST/ALT on AKI development after CABG. Acute kidney injury was observed in $15.8 \%$ of the patients in our study. Postoperative AKI occurred in 27 patients in Group I and 13 patients in Group II. Logistic regression analysis, revealing the higher AST/ALT preoperatively (T0), was shown to be associated with an increased incidence of postoperative AKI (OR, 3.983; 95\% CI, 1.940-8.180; $P<.001)$. Additionally, higher AST/ALT on the postoperative day 1 (T1) and postoperative day 3 (T2) were reported to be associated with an increased incidence of postoperative AKI ( $P=.004$ and $P=.015$, respectively).

AST is mainly found in mitochondria of liver cells, however, it also is present in the heart, skeletal muscle, lungs, brain, and kidneys [Woreta 2014]. ALT is found only in the liver. AST/ALT is widely used for assessment of severity of liver injury. In addition to this use, it also is used in extrahepatic diseases, such as assessment of prognosis of cancer or prediction of cardiovascular events [Gurung 2013; Huang 2017]. One of the main findings in the present study is the increased AST/ALT preoperatively. An explanation to this finding could be the extrahepatic release of AST. As suggested above, AST also is present in other tissues, mainly released from myocardial cells, and myocardial injury preoperatively could lead to an increase in AST/ALT [Ewid 2020]. Another important finding of the study is the association of both preoperative and postoperative high AST/ALT with postoperative AKI. Renal dysfunction after cardiac surgery is a common complication; however, the exact mechanism still is unknown. Hypertension, DM, age, severe arteriosclerosis, and impaired left ventricular function are the known major preoperative risk factors for postoperative AKI [Doddakula 2007]. The intraoperative causative mechanisms of AKI during cardiac surgery are CPB induced SIRS that results in the production of excessive ROS, ischemia-reperfusion that also leads to the generation of free oxygen radicals and hypoperfusion of the organs, mainly the kidneys and liver. Serum AST and ALT are inflammation-associated components, and it is known that free radicals, microembolism and inadequate tissue perfusion are related to both hepatic dysfunction and AKI after cardiopulmonary bypass [Di Tomasso 2015], and these effects of $\mathrm{CPB}$ could explain the relation of AST/ALT and AKI after CABG in our study.

In our study, there was a significant gender difference between groups that females showed a higher AST/ALT preoperatively, and this result was compatible with the literature [Lin 2019]. Our results showed that metabolic syndrome (MetS) was more common in patients with lower AST/ALT than in the higher ratio group. MetS is a common problem in the general population and also in patients with cardiovascular disease. There is an ongoing debate about the link between AST/ALT and MetS. It is reported that lower AST/ ALT was associated with MetS, and it is suggested that the AST/ALT would be a useful, simple, and effective serum marker of MetS [Lu 2012]. Obesity (BMI $>30 \mathrm{~kg} / \mathrm{m}^{2}$ ), also a component of MetS, was reported to be more common in lower AST/ALT group in our study. It is suggested that 
patients with $\mathrm{BMI}>30 \mathrm{~kg} / \mathrm{m}^{2}$ were higher in patients with a lower AST/ALT, which denotes a correlation of AST/ALT and MetS may be mediated by abdominal obesity [Xiaoli 2012]. Liver enzymes are used as biomarkers of non-alcoholic fatty liver, and ALT is used for a screening blood test in obese children for prediction of fatty liver [Elizondo-Montemayor 2014]. As fatty liver is known to be associated with abdominal obesity, it is plausible that the AST/ALT was lower in patients with $\mathrm{BMI}>30 \mathrm{~kg} / \mathrm{m}^{2}$ in the present study. In the literature, it is suggested that DM, MetS and obesity (BMI $\left.>30 \mathrm{~kg} / \mathrm{m}^{2}\right)$ are associated with postoperative AKI after CABG [Tekeli Kunt 2016; Heringlake 2016]. In the present study, multivariate regression analysis revealed that DM, obesity and MetS were shown to be independent risk factors for AKI after CABG (OR, 3.348; 95\% CI, 1.650-6.799; $P=.001$, OR, 2.885; 95\% CI, 1.435-5.798; $P<.001$, OR, 4.195; 95\% CI, 2.068-8.509; $P=.003$, respectively).

We compared the patients according to the severity of AKI and reported that Group I, in which patients had higher levels of preoperative AST/ALT, had a higher number of patients in RIFLE I and RIFLE F stages and higher levels of postoperative (T1 and T2) AST/ALT had a higher number of patients in RIFLE F stage. The putative mechanisms underlying the relationship between increased AST/ALT and AKI are not well understood. We postulate that oxidative stress may play a role as both inflammation and oxidative stress increase intracellular calcium levels that results in intrarenal vasoconstriction and decrease in renal blood flow leading to acute kidney injury [Botezelli 2012; Xu 2019]. It is suggested that postoperative AKI requiring RRT has an overall mortality of $37-60 \%$. It has an independent effect on early mortality and morbidity and also the quality of life in the short- and long-term. Continuous RRT aims to treat AKI by maintaining hemodynamic stability, acid-base, and electrolyte balances in critically ill patients with a very high risk of mortality [Huen 2012]. The present study demonstrates that RRT was required in 1.98\% of patients, and all patients had higher AST/ALT. None of the patients became hemodialysis dependent. There is not a consensus regarding the timing and type of RRT to apply, the decisions regarding these factors were made together with our nephrologists in this study [Kunt 2009; Demirkıllıç 2004].

The mean ICU time, length of hospital stay, and mean ventilatory support times were statistically longer in Group I. This could be due to the increased use of RRT in these patients. Postoperative renal dysfunction affects not only mortality but also quality of life. AKI plays a vital role in postoperative mortality and morbidity in cardiac surgery patients. The rate of RRT is suggested to be $5 \%$ and $6 \%$ in patients with a high prevalence of AKI [Uchino 2005]. Among the patients who developed AKI, RRT was applied to $18.5 \%$ of the patients in our study group with higher AST/ALT and none in lower AST/ALT group $(P=.009)$. The overall 30-day mortality was $3.6 \%(\mathrm{~N}=9)$ seven patients in Group I and two patients in Group II, where the difference was statistically significant. All of the patients died, due to low cardiac output and required RRT.

The main strength of the study was that, to our knowledge, this is the first study to report the predictive effect of both preoperative and postoperative AST/ALT on postoperative AKI in patients who underwent cardiac surgery. There also are some limitations of the study. First, the study design is retrospective. Second, the literature studies - either clinical or experimental - that focus on the relation between AST/ ALT and AKI are limited.

\section{CONCLUSION}

AKI after CABG results in various postoperative complications and leads to prolonged hospitalization, increased costs, and increased rate of mortality. A preoperative AST/ ALT of 1.22 was the best cutoff point for predicting postoperative AKI in the present study. Preoperative and postoperative AST/ALT might be an independent predictor of AKI after cardiac surgery.

\section{REFERENCES}

Bellomo R, Ronco C, Kellum JA, et al. 2004. Acute Dialysis Quality Initiative workgroup. Acute renal failure - definition, outcome measures, animal models, fluid therapy and information technology needs: the Second International Consensus Conference of the Acute Dialysis Quality Initiative (ADQI) Group. Crit Care. 8:204-12.

Botezelli JD, Cambri LT, Ghezzi AC, et al. 2012. Fructose-rich diet leads to reduced aerobic capacity and to liver injury in rats. Lipids Health Dis. 11:78-84.

Botros M, Sikaris KA. 2013. The de Ritis ratio: the test of time. Clin Biochem Rev. 34:117-30.

Brown JR, Cochran RP, Leavitt BJ, et al. 2007. Multivariable prediction of renal insufficiency developing after cardiac surgery. Northern New England Cardiovascular Disease Study Group. Circulation. 116:139-43.

Chertow GM, Lazarus JM, Christiansen CL, et al. 1997. Preoperative renal risk stratification. Circulation. 95:878-84.

Cockcroft DW, Gault MH. 1976. Prediction of creatinine clearance from serum creatinine. Nephron. 16:31-41.

Demirkılıç U, Kuralay E, Yenicesu M, et al. 2004. Timing of replacement therapy for acute renal failure after cardiac surgery. J Card Surg. 19:17-20.

Di Tomasso N, Monaco F, Landoni G. 2015. Hepatic and renal effects of cardiopulmonary bypass. Best Pract Res Clin Anaesthesiol. 29(2):151-61.

Doddakula K, Al-Sarraf N, Gately K, et al. 2020. Predictors of acute renal failure requiring renal replacement therapy post cardiac surgery in patients with preoperatively normal renal function. Interact Cardiovasc and Thorac Surg. 6:314-8.

Elizondo-Montemayor L, Ugalde-Casas PA, Lam-Franco L, et al. 2014. Association of ALT and the metabolic syndrome among Mexican children. Obes Res Clin Pract. 8(1):79-87.

Ewid M, Sherif H, Allihimy AS, et al. 2020. AST/ALT ratio predicts the functional severity of chronic heart failure with reduced left ventricular ejection fraction. BMC Res Notes;13:178-84.

Gao F, Chen C, Lu J, et al. 2017. De Ritis Ratio (AST/ALT) as an Independent Predictor of Poor Outcome in Patients With Acute Ischemic Stroke. Neuropsychiatr Dis Treat. 13:1551-7. 
Gurung RB, Purbe B, Gyawali P, et al. 2013. The ratio of aspartate aminotransferase to alanine aminotransferase (AST/ ALT): the correlation of value with underlying severity of alcoholic liver disease. Kathmandu University Medical Journal. 11(43):233-6.

Hall RI, Smith MS, Rocker G. 1997. The systemic inflammatory response to cardiopulmonary bypass: pathophysiological, therapeutic, and pharmacological considerations. Anesth Analg. 85:766-82.

Heringlake M, Charitos EI, Erber K, et al. 2016. Preoperative plasma growth-differentiation factor-15 for prediction of acute kidney injury in patients undergoing cardiac surgery. Crit Care. 20(1):317-27.

Huang H, Wang XP, Li XH, et al. 2017. Prognostic value of pretreatment serum alanine aminotransferase/aspartate aminotransferase (ALT/AST) ratio and gamma glutamyltransferase (GGT) in patients with esophageal squamous cell carcinoma. BMC Cancer. 17(1):544-55.

Huen S, Parikh CR. 2012. Predicting acute kidney injury following cardiac surgery: a systematic review. The Annals of Thoracic Surgery. 93:337-47.

Isbir SC, Tekeli A, Ergen A, et al. 2007. Genetic polymorphisms contribute to acute kidney injury after coronary artery bypass grafting. Heart Surg Forum. 10:439-44.

Kunt AT, Akgün S, Atalan S, et al. 2009. Furosemide infusion prevents the requirement of renal replacement therapy after cardiac surgery. Anadolu Kardiyol Derg. 9:499-504.

Lassnigg A, Donner E, Grubhofer G, et al. 2000. Lack of renoprotective effects of dopamine and furosemide during cardiac surgery. J Am Soc Nephrol. 11:97-104.

Lin S, Tang L, Jiang R, et al. 2019. The Relationship Between Aspartate Aminotransferase To Alanine Aminotransferase Ratio And Metabolic Syndrome In Adolescents In Northeast China. Diabetes Metab Syndr Obes. 12:2387-94.

Lu Q, Liu X, Liu S, et al. 2012. The relationship between AST/ALT ratio and metabolic syndrome in Han young adults - AST/ALT ratio and metabolic syndrome. In: Mehnaz A editor, Recent advances in cardiovascular risk factors. InTech. 247-54.
Rief P, Pichler M, Raggam R, et al. 2016. The AST/ALT (De-Ritis) ratio: A novel marker for critical limb ischemia in peripheral arterial occlusive disease patients. Medicine. 95(24):3843-7.

Sheth SG, Flamm SL, Gordon FD, et al. 1998. AST/ALT ratio predicts cirrhosis in patients with chronic hepatitis $\mathrm{C}$ virus infection. The American journal of gastroenterology. 93(1):44-8.

Steininger M, Winter MP, Reiberger T, et al. 2018. De-Ritis Ratio Improves Long-Term Risk Prediction After Acute Myocardial Infarction. J Clin Med. 7(12):474-86.

Suen WS, Mok CK, Chiu SW, et al. 1998. Risk factors for development of acute renal failure (ARF) requiring dialysis in patients undergoing cardiac surgery. Angiology. 49:789-800.

Tekeli Kunt A, Parlar H, Findik O, et al. 2016. The Influence of Metabolic Syndrome on Acute Kidney Injury Occurrence after Coronary Artery Bypass. Heart Surg Forum. 19:099-103.

Uchino S, Kellum JA, Belloma R, et al. 2005. Acute renal failure in critically ill patients; a multinational, multicenter study. JAMA. 294:813-8.

Weerasinghe A, Hornick P, Smith P, et al. 2001. Coronary artery bypass grafting in non- dialysis-dependent mild-to-moderate renal dysfunction. J Thorac Cardiovasc Surg. 121:1083-9.

Woreta TA, Alqahtani SA. 2014. "Evaluation of abnormal liver tests," Medical Clinics of North America. 98(1):1-16.

Xiaoli LIU, Qiang LU, Junfeng JIAO, et al. 2012. Relationship between aspartate aminotransferase to alanine aminotransferase ratio and metabolic syndrome in college students. Chinese Journal of Health Management. 6:409-412.

Xu J, Xia Y, Li S, et al. 2019. A retrospective pilot study to examine the potential of aspartate aminotransferase to alanine aminotransferase ratio as a predictor of postoperative acute kidney injury in patients with hepatocellular carcinoma. Ann Clin Biochem. 56(3):357-66.

Yeo KK, Li Z, Yeun JY, et al. 2008. Severity of chronic kidney disease as a risk factor for operative mortality in nonemergent patients in the california coronary artery bypass graft surgery outcomes reporting program. Am J Cardiol. 101:1269-74. 